\title{
Towards a temperature dependent and probabilistic lifetime concept for nodular ductile cast iron materials undergoing isothermal and thermo-mechanical fatigue
}

\author{
Elena Garcia Trelles ${ }^{1, *}$, Christoph Schweizer $^{1}$, and Stefan Eckmann ${ }^{1}$ \\ ${ }^{1}$ Fraunhofer Institute for mechanics of materials IWM, Wöhlerstraße 11, 79108 Freiburg im Breisgau, Germany
}

\begin{abstract}
In this investigation, the fatigue behaviour of a ductile cast iron with high content of silicon and molybdenum, was experimentally characterized by performing isothermal low cycle fatigue (LCF) tests as well as out-of-phase thermomechanical fatigue (OPTMF) tests within the temperature range RT $-500{ }^{\circ} \mathrm{C}$. The studied material shows an embrittlement at temperatures nearby $400{ }^{\circ} \mathrm{C}$. A possible explanation for the observed lifetime reduction is intergranular embrittlement (IE). A mechanism based lifetime model is proposed for assessing the lifetime. The model is based on the assumption that the crack advance per cycle is correlated with the cyclic crack tip opening displacement ( $\triangle \mathrm{CTOD}$ ) attributed to the crack tip blunting caused by accumulation of plastic and creep deformations ahead of the crack tip. Intergranular embrittlement is accounted for by introducing a temperature and strain rate dependent prefactor in the crack growth law, which only acts in a certain temperature range. The model is calibrated for a GJS material and successfully applied to predict the lifetime of this material when undergoing isothermal and non-isothermal mechanical loadings. A probabilistic interpretation of the scatter of the investigated material is presented in conjunction with the random nature of the initial defect size distribution.
\end{abstract}

\section{Introduction}

Nodular ductile cast iron materials (DCI) provide a good performance at high temperatures, especially DCI materials with high content of silicon and molybdenum. Due to their good mechanical properties, castability and cost efficient production processes, the use of these materials for fabricating components undergoing thermomechanical fatigue (i.e. cylinder heads, turbochargers, motor housings) is widely spread. In order to guarantee the safety of these components the damage mechanisms involved in the crack initiation and propagation regimes must be carefully investigated and integrated into lifetime concepts.

For non-isothermal conditions, the time and temperature dependent damage parameter $\mathrm{D}_{\mathrm{TMF}}$ [1] was successfully applied for describing the fatigue damage of numerous high temperature resistant alloys. This parameter accounts for the temperature dependent material properties, the cyclic stresses and plastic strains, fatigue crack closure as well as for creep accelerated fatigue crack growth. The lifetime assessment is performed by assuming that the crack growth per cycle is proportional to the crack tip opening displacement.

However, this model formulation does not account for IE damage. The presence of IE damage is related to a shorter fatigue lifetime and the modelling of DCI materials suffering from IE damage demands a new formulation.
The IE damage mechanism was studied by several authors [2-6]. Nevertheless there is no clear explanation for this phenomenon. It is known to be dependent on microstructural features, such as the graphite size and the eutectic cell wall size, where greater sizes should promote intergranular fracture [2]. Based on tensile testing, it was also studied in [3] how increasing the percentage of silicon, changes the fracture mechanism from a mix of dimpled and intergranular fracture to cleavage and intergranular fracture. This change of fracture mechanism is thought to be related with the presence of $\mathrm{MgO}$ particles in the eutectic cell wall region serving as crack initiation sites [4]. Another investigation [5] concludes that $\mathrm{MgO}$ inclusions serve as crack initiation sites and the amount of phosphorus segregation on the grain boundaries dictates the fracture mode within the IE temperature of ferritic DCIs. In the same study the amount of segregated phosphor is connected to the solidification cooling rates where slower cooling rates should enhance phosphorus segregation. The kinematics of the process seem to be unclear, however all authors observed a drop in the ductility of the material at the IE temperature for the conducted tensile tests. [6] observed that the ductility drop derived from the IE damage is also dependent on the strain rate of the performed tensile tests. In this work the IE damage was modelled based on tensile tests performed at different temperatures and strain rates including the effect of strain rate on the IE damage process. [6] and [7] investigated the strain rate 
dependency of the IE effect, concluding that increasing strain rates shift the IE temperature (corresponding to the ductility minimum) to higher temperature values

The first aim of this study is to propose a lifetime model including the damaging effect of IE to enable reliable lifetime assessment of DCIs. This is achieved with the introduction of the IE damage factor from [6] in the mechanism based damage model from [1] by modifying the original model formulation based on the $\mathrm{D}_{\mathrm{TMF}}$ parameter in order to account for IE damage, which is expected to lead to accelerated crack growth and reproduces the temperature dependent lifetime reduction. The second aim is to validate the model through the LCF and OPTMF performed tests with the ferritic DCI GJS.

A probabilistic concept is introduced in order to provide an intuitive interpretation of the material scatter, attributing this inherent scatter to the fact that the initial flaw size causing crack initiation has not a fixed size. A more realistic approach to real conditions is to work with an initial flaw size probability distribution function.

\section{Experimental}

The ductile cast iron material concerning this study is referred as GJS due to confidentiality reasons. The microstructure of this spheroidal cast iron with ferritic matrix is illustrated in Fig. 1.

For characterizing the mechanical behaviour of the cast iron GJS, tensile tests, strain controlled LCF tests and OPTMF tests were carried out for representative strain amplitudes within the low cycle fatigue regime.

The tensile tests were performed at RT, $100{ }^{\circ} \mathrm{C}$, $200{ }^{\circ} \mathrm{C}, 300{ }^{\circ} \mathrm{C}, 350{ }^{\circ} \mathrm{C}, 400{ }^{\circ} \mathrm{C}, 450{ }^{\circ} \mathrm{C}$ and $500{ }^{\circ} \mathrm{C}$ with a strain rate of $\dot{\varepsilon}=10^{-4} \mathrm{~s}^{-1}$. Three further tensile tests were performed at $450{ }^{\circ} \mathrm{C}$ and one at $500{ }^{\circ} \mathrm{C}$ with $\dot{\varepsilon}=10^{-3} \mathrm{~s}^{-1}$ in order to study the strain rate dependency of the mechanical properties.

The tensile tests were conducted with an electromechanical tensile testing machine Kappa SS from Zwick Roell. The specimens were heated using a three-zone radiation furnace from MTS. Type K thermocouples were used.

LCF tests were carried out at RT, $300{ }^{\circ} \mathrm{C}, 350{ }^{\circ} \mathrm{C}$, $400{ }^{\circ} \mathrm{C}, 450{ }^{\circ} \mathrm{C}$ and $500{ }^{\circ} \mathrm{C}$ with $\dot{\varepsilon}=10^{-3} \mathrm{~s}^{-1}$ to characterize the fatigue behaviour within the temperature range $\mathrm{RT}-500{ }^{\circ} \mathrm{C}$.

OPTMF tests were performed to study the thermomechanical behaviour of GJS. For the thermal cycle a minimum temperature equal to $50{ }^{\circ} \mathrm{C}$ was fixed for all the specimens and the maximum temperature of the thermal cycles was set to $350{ }^{\circ} \mathrm{C}, 400{ }^{\circ} \mathrm{C}, 450{ }^{\circ} \mathrm{C}$ and $500{ }^{\circ} \mathrm{C}$. The tests were conducted with a fixed heating rate of $5^{\circ} \mathrm{C} / \mathrm{s}$ and air cooling was applied during cooling of the specimens. To study if the material exhibits time dependent behaviour, two OPTMF tests were conducted with a dwell time of $300 \mathrm{~s}$ applied at the minimum strain (dwell time in compression).

The LCF and OPTMF tests were performed on an Instron 8862 electromechanical testing machine. The specimens were heated using a high frequency induction heating system. Temperature control was achieved using type $\mathrm{K}$ thermocouples.

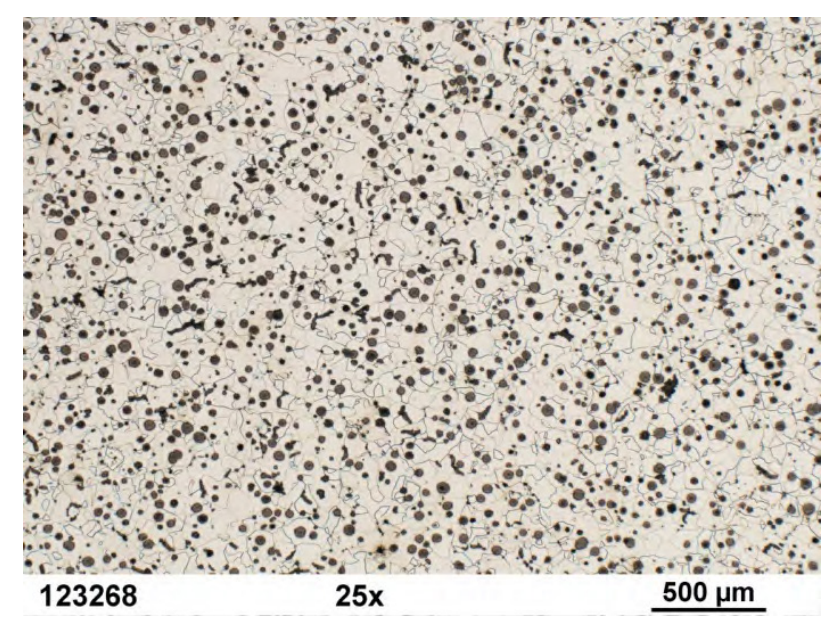

Fig. 1. Optical micrograph of the material GJS etched for $6 \mathrm{~s}$ with $3 \%$ Nital.

\section{Results and discussion}

\subsection{Monotonic material behaviour}

In this section, the monotonic material properties evaluated from the conducted tests with $\dot{\varepsilon}=10^{-4} \mathrm{~s}^{-1}$ and $\dot{\varepsilon}=10^{-3} \mathrm{~s}^{-1}$ are presented in Fig. 2. It is observed that there is no significant strain rate effect on the tensile strength and monotonic yield stress within the studied temperature range. However, Fig. 3 illustrates that the temperature corresponding to the ductility minimum is dependent on the strain rate applied of the tensile test. It is observed how increasing the strain rate of the experiment, increases the temperature at which the ductility drop takes place. This agrees with the afore mentioned investigations from $[6,7]$.

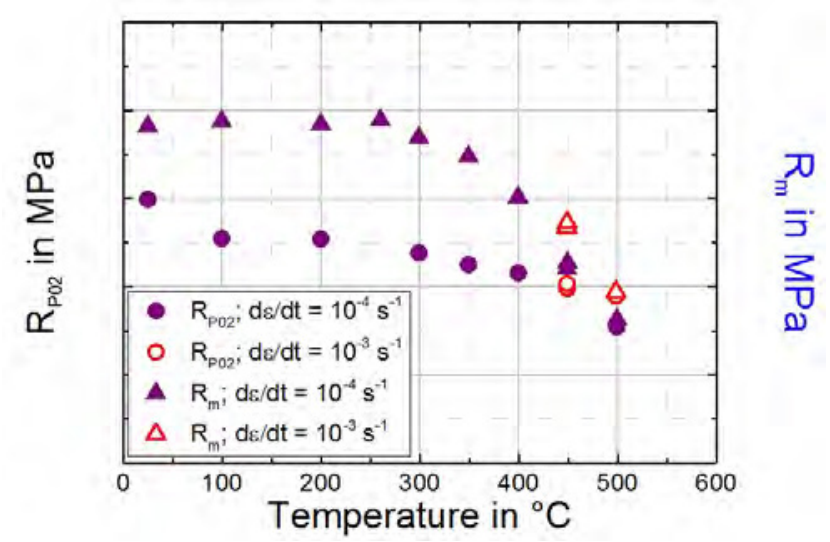

Fig. 2. Tensile strength and monotonic yield stress over temperature. 


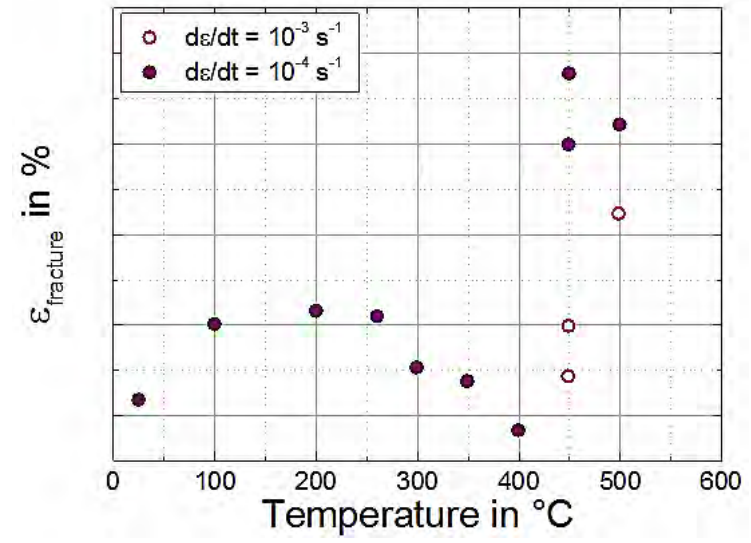

Fig. 3. Fracture strain over temperature.

\subsection{Cyclic material behaviour}

The cyclic material behaviour is investigated based on the LCF and OPTMF tests.

Based on the midlife cycle of each LCF test, an averaged Young's modulus, cyclic yield stress and hardening exponent were obtained for each investigated temperature. The Young's modulus decreases approximately linearly over temperature. The values of the cyclic yield stress over temperature stay relatively constant until $300{ }^{\circ} \mathrm{C}$, dropping approximately linearly from this temperature until $500^{\circ} \mathrm{C}$. The RambergOsgood hardening exponent is almost three times higher at RT with approximately constant values between $300{ }^{\circ} \mathrm{C}$ and $500{ }^{\circ} \mathrm{C}$.

The Wöhler curves of GJS illustrated in Fig. 4, point to lifetimes ten times shorter for the temperature range 400-450 ${ }^{\circ} \mathrm{C}$ in comparison to the lifetimes of tests conducted with similar strain amplitudes at RT and $500{ }^{\circ} \mathrm{C}$. This temperature range coincides with the temperature range in Fig. 3, where the ductility drop is observed for the tensile tests conducted with $\dot{\varepsilon}=$ $10^{-3} \mathrm{~s}^{-1}$. For $\varepsilon_{\mathrm{a} \text {,mech }}=0.5 \%$ an LCF test at $450{ }^{\circ} \mathrm{C}$ and two OPTMF tests conducted with a maximum temperature equal to $500{ }^{\circ} \mathrm{C}$. It is observed that the OPTMF tests show significantly longer lifetimes than the corresponding LCF test. By comparing the two OPTMF tests, one performed with an applied dwell time of $300 \mathrm{~s}$ and the other without, it can be stated that no time dependent behaviour of the GJS material in the temperature range $\mathrm{RT}-500^{\circ} \mathrm{C}$ is observed.

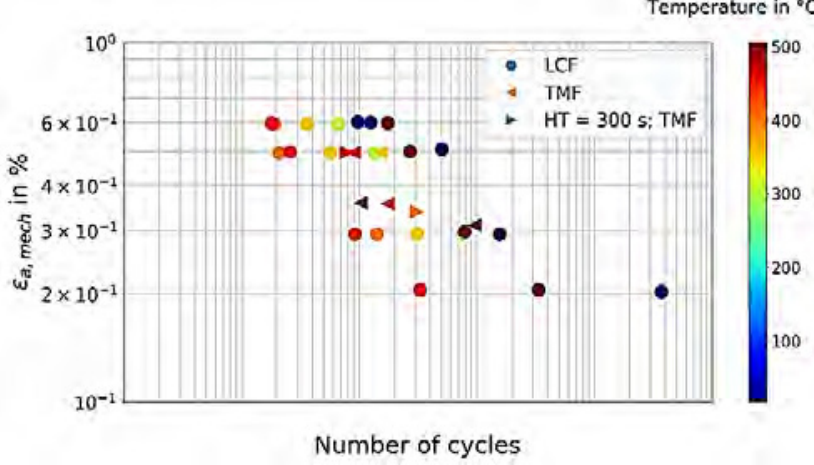

Fig. 4. Fatigue lives of LCF and OPTMF.

\subsection{Lifetime model}

\subsubsection{Uniaxial lifetime model formulation and calibration}

In this section a lifetime model including the main damage mechanisms causing fatigue failure is formulated.

For non-isothermal conditions, the time and temperature dependent damage parameter $\mathrm{D}_{\mathrm{TMF}}$ [1] has been successfully applied for describing the fatigue damage of numerous high temperature resistant alloys. This parameter accounts for the temperature dependent material properties, the cyclic stresses and plastic strains, fatigue crack closure as well as for creep accelerated fatigue crack growth. The lifetime assessment is performed by assuming that the crack growth per cycle is proportional to the cyclic crack tip opening displacement as described in Eq.(1):

$$
\frac{d a}{d N_{\text {fat }}}=\beta \triangle C T O D^{B} .
$$

The cyclic crack tip opening displacement, $\triangle \mathrm{CTOD}$, is calculated by means of fracture mechanics. For an elastic plastic material and a semicircular surface crack under monotonic loading, the J-Integral is calculated by [8] as:

$$
J=\left(1.45 \frac{\sigma^{2}}{E}+2.4 \frac{\sigma \varepsilon^{p l}}{\sqrt{1+3 N^{\prime}}}\right) a .
$$

The first term of Eq. (2) accounts for the elastic contribution and the second term for the plastic contribution. $\sigma$ is the stress, $\varepsilon^{\mathrm{pl}}$ is the plastic deformation and the parameter $\mathrm{N}^{\prime}$ is the Ramberg-Osgood hardening exponent.

The crack tip opening displacement and the J-Integral are related by [9]:

$$
C T O D=d_{N^{\prime}} \frac{J}{\sigma_{y}}
$$

Where $\sigma_{\mathrm{y}}$ denotes the yield stress and $\mathrm{d}_{\mathrm{N}}$, depends on the hardening behavior of the material. For power-law hardening materials, the crack-tip fields are the Hutchinson, Rice and Rosengren (HRR) singular fields [10]. For cyclic loading, the parameters in Eqs. (2) and (3) are replaced by their cyclic counterparts and $\triangle$ CTOD is computed as:

$$
\triangle C T O D=d_{N^{\prime}} \frac{\Delta J_{e f f}}{\sigma_{c y}} .
$$

$\sigma_{c y}$ is the cyclic yield stress of the material and it is evaluated for a stabilized cycle. For a small semicircular surface crack, the cyclic J-integral in Eq. (4) can be estimated from the damage parameter according to Heitmann [11]:

$$
Z_{D}=\left(1.45 \frac{\Delta \sigma_{e f f}^{2}}{E}+2.4 \frac{\Delta \sigma \Delta \varepsilon^{p l}}{\sqrt{1+3 N^{\prime}}}\right)
$$

Thus, Eq. (4) can be rewritten as:

$$
\triangle C T O D=d_{N \prime} \frac{Z_{D}}{\sigma_{c y}} a .
$$

This is equivalent to the expression:

$$
\triangle C T O D=\frac{d_{N^{\prime}}}{\sigma_{c y}}\left(1.45 \frac{\Delta \sigma_{e f f}{ }^{2}}{E}+2.4 \frac{\Delta \sigma \Delta \varepsilon^{p l}}{\sqrt{1+3 N^{\prime}}}\right) a,
$$


where $\mathrm{N}^{\prime}$ is the hardening exponent, obtained by fitting the Ramberg Osgood equation (Eq. 8) to a stabilized cycle.

$$
\Delta \varepsilon=\frac{\Delta \sigma}{E}+0.002\left(\frac{\Delta \sigma}{\sigma_{c y}}\right)^{\frac{1}{N^{\prime}}}
$$

The index eff implies that crack closure effects are included. In Eqs. (5) and (7), $\Delta \sigma$ and $\Delta \varepsilon^{\mathrm{pl}}$ denote the stress range and the plastic strain range and can be taken from a stabilized hysteresis loop, e.g. the mid-life hysteresis loop.

Crack closure is accounted by the use of the effective stress range:

$$
\Delta \sigma_{e f f}=\sigma_{\max }-\sigma_{o p} .
$$

$\sigma_{\max }$ is the maximum stress and can be determined from the stress-strain hysteresis loops as well. $\sigma_{\text {op }}$ is the crack opening stress. The crack opening stress can be determined from empirical formulas or numerical calculations. In order to calculate the effective stress range, Schijve [9] and Schmitt [12] proposed the equation:

$$
\Delta \sigma_{e f f}=\Delta \sigma U\left(R_{\sigma}\right)
$$

The function $\mathrm{U}\left(\mathrm{R}_{\sigma}\right)$ considers the load ratio dependency. The Newman crack closure model was chosen for modelling the investigated material. The main reason for this selection is the fact that in contrast with other empirical expressions available for modelling crack closure effects Newman proposed a dependency on the maximum stress, which was derived from systematic investigations with the strip yield model [13]:

$$
U\left(R_{\sigma}, \sigma_{\max }\right)=\frac{1-\frac{\sigma_{o p}}{\sigma_{\max }}}{1-R_{\sigma}} .
$$

In Eq. (11), the term $\frac{\sigma_{\mathrm{op}}}{\sigma_{\max }}$ is calculated with the help of the polynomial functions given in Eqs. (12) and (13):

$$
\begin{gathered}
\frac{\sigma_{o p}}{\sigma_{\max }}=A_{0}+A_{1} R_{\sigma}+A_{2} R_{\sigma}{ }^{2}+A_{3} R_{\sigma}^{3} \text { for } \\
R_{\sigma} \geq 0, \\
\frac{\sigma_{o p}}{\sigma_{\max }}=A_{0}+A_{1} R_{\sigma} \text { for }-1 \leq R_{\sigma}<0, \\
A_{0}= \\
\left(0.825-0.34 \alpha+0.05 \alpha^{2}\right)\left[\operatorname { c o s } \left(\pi \sigma_{\max } /\right.\right. \\
\left.\left.\left(2 \sigma_{y}\right)\right)\right]^{\frac{1}{\alpha},} \\
A_{1}=(0.415-0.071 \alpha) \sigma_{\max } / \sigma_{y}, \\
A_{2}=1-A_{0}-A_{1}-A_{3}, \\
A_{3}=2 A_{0}+A_{1}-1,
\end{gathered}
$$

where $\alpha=1$ for plain stress and $\alpha=3$ for plain strain. Newman's formula tends to predict decreasing opening stresses with increasing maximum stresses, which is in accordance with experimental observations.

A generalization of Eq. (6) for elastic-viscoplastic material behaviour under cyclic loading is achieved by the $\mathrm{D}_{\mathrm{TMF}}$ damage parameter [12] and leads to the equation below:

$$
\triangle C T O D=d_{N^{\prime}} D_{T M F} a .
$$

$\mathrm{D}_{\mathrm{TMF}}$ is defined by the following equation:

$$
D_{T M F}=\frac{Z_{D}}{\sigma_{c y}} F(t, \sigma, T) .
$$

The function $F(t, \sigma, T)$ describes the time dependent effects, which are a function of the loading history. $\mathrm{F}$ is defined as:

$$
\begin{aligned}
F(t, \sigma, T)=[1+ & \alpha^{\prime} \\
& \quad \exp \left(\frac{Q_{c r}}{R T_{0}}\right) \int_{t_{\text {start }}}^{t_{\text {end }}} \sigma_{c y}^{n-2} * \delta \sigma \\
& \left.\quad \exp \left(-\frac{Q_{c r}}{R T}\right) d t\right]^{\frac{1}{n}}
\end{aligned}
$$

Where $\mathrm{n}$ is the Norton creep exponent, $\mathrm{Q}_{\mathrm{cr}}$ is the activation energy, $\mathrm{R}$ is the universal gas constant, $\mathrm{T}_{0}$ a reference temperature (typically $\mathrm{T}_{0}=227^{\circ} \mathrm{C}$ ). The parameter $\alpha^{\prime}$ is an adjustable parameter.

The symbol " $\delta$ " denotes the stress increment in Eq. (20):

$$
\delta \sigma=\sigma(t)-\sigma\left(t_{\text {start }}\right)
$$

Here, $\mathrm{t}_{\text {start }} \leq \mathrm{t} \leq \mathrm{t}_{\text {end }}$ holds. $\mathrm{t}_{\text {start }}$ and $\mathrm{t}_{\text {end }}$ denote the start and the end of the analyzed branch of the loading cycle.

For TMF loading, all temperature dependent material properties such as $\sigma_{\mathrm{cy}}, \mathrm{E}$ and N' are taken as the average of their minimum and maximum values occurring within a branch (loading or unloading).

In order to receive an expression for the lifetime, it is assumed that the (short) crack growth phase dominates the lifetime. Inserting Eq. (18) into Eq. (1) one obtains:

$$
\frac{d a}{d N_{\text {fat }}}=\beta\left(d_{N^{\prime}} D_{T M F} a\right)^{B},
$$

where $\beta$ and $B$ are material dependent parameters.

The crack tip opening displacement is calculated based on the $\mathrm{D}_{\text {TMF }}$ parameter by integrating the damage over the crack length for an initial crack size $a_{0}$ and a technical final crack size $a_{\mathrm{f}}$. The number of cycles consumed by fatigue damage takes the form:

$$
N_{f a t}=\frac{a_{f}^{1-B}-a_{0}^{1-B}}{\beta *(1-B)} *\left(d_{N^{\prime}} D_{T M F}\right)^{-B} .
$$

However, the $\mathrm{D}_{\mathrm{TMF}}$ parameter does not always lead to satisfying lifetime correlations for DCI materials under low cycle and thermomechanical fatigue loading as depicted in Fig. 5, because it does not account for an embrittlement effect at intermediate temperatures. Hence, no temperature independent picture is obtained in Fig. 5 due to an underestimated damage contribution at temperatures close to the intergranular embrittlement temperature. With this motivation and the work from [6] in mind, the crack growth model based on $\mathrm{D}_{\mathrm{TMF}}$ is modified to account for the embrittlement effect by integrating a temperature dependency into the parameter $\beta$ from the crack growth law. 


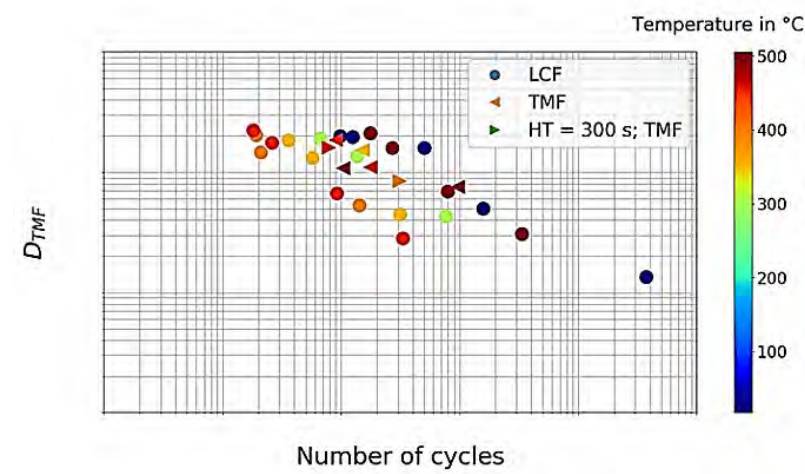

Fig. 5. $\mathrm{D}_{\mathrm{TMF}}$ versus experimental lifetimes.

Kobayashi et al. [7] studied the ductility drop at the IE (intergranular embrittlement) temperature, which is thought to be related to the lifetime reduction of fatigue tests performed at temperatures near to the IE temperature [14]. He observed that the IE temperature (with the corresponding ductility drop) shifted to higher temperatures for tensile tests conducted with higher strain rates following an Arrhenius relationship, such that:

$$
\dot{\varepsilon}=A_{I E} e^{-\frac{Q_{I E}}{R T_{m}}} .
$$

$\mathrm{T}_{\mathrm{m}}$ is the IE temperature corresponding to the strain rate applied for conducting the tensile test and $\mathrm{R}$ the universal gas constant. For modelling the IE damage of GJS, the activation energy was set to the value proposed in [6], $Q_{I E}=290000 \mathrm{KJ} / \mathrm{mol}$, and $A_{I E}$ was indirectly adjusted in order to match the experimentally observed IE temperature in Fig. 3, leading to $\mathrm{A}_{\mathrm{IE}}=2 * 10^{19} \mathrm{~s}^{-1}$.

In [6], $\mathrm{Wu}$ et al. introduced a damage factor for considering the damage caused by intergranular embrittlement, with the following form:

$$
D_{I E}=1+\frac{l}{\lambda} \varphi(T)
$$

where $\Phi(\mathrm{T})$ is the temperature dependent IE damage function and $\Phi(T) \epsilon[0,1]$. The IE damage function can be modelled by means of a Gaussian function, whose parameters can be calibrated by fitting the Gauss function to the normalized rupture strain variation over temperature from tensile tests performed at different temperatures. This function relates the reduction of ductility over temperature with the lifetime reduction. The parameter l represents the eutectic cellular size in DCIs and $\lambda$ the grain boundary Mo-rich eutectic cell thickness (from [6]: $\mathrm{l}=100 \mu \mathrm{m}$ and $\lambda$ varies between 2 and $7 \mu \mathrm{m}) \cdot \frac{1}{\lambda}$ weights the effect of the IE damage. If $\varphi(\mathrm{T})=0$, there is no IE damage acting, whereas for $\varphi(\mathrm{T})=1$ Eq. (25) reaches its maximum value.

The advantage of describing the IE damage with a Gaussian function is that the maximum IE damage takes place at the IE temperature $\left(\mathrm{T}_{\mathrm{m}}\right)$, therefore $\varphi\left(\mathrm{T}_{\mathrm{m}}\right)=1$. On the other hand the center of symmetry of the Gauss function coincides with the IE temperature, which can be recalculated for different strain rates using Eq. (24) and thus, shift the $\varphi(\mathrm{T})$ function consequently in order to recalculate the IE damage for the specific strain rate and temperature.

$$
\varphi(T)=e^{-\frac{\left(T-T_{m}\right)^{2}}{w^{2}}}
$$

The only parameter left to calibrate is $\mathrm{w}$, which gives a measure of the width of the adjusted Gaussian function, a broad bell shape would imply that the IE damage is present over a wider range of temperatures surrounding $\mathrm{T}_{\mathrm{m}}$, a narrow bell would suggest that the temperature interval where IE damage is acting, is smaller. Fig. 6 shows the calibrated IE damage function for GJS. For higher strain rates, $\mathrm{T}_{\mathrm{m}}$ shifts to higher values.

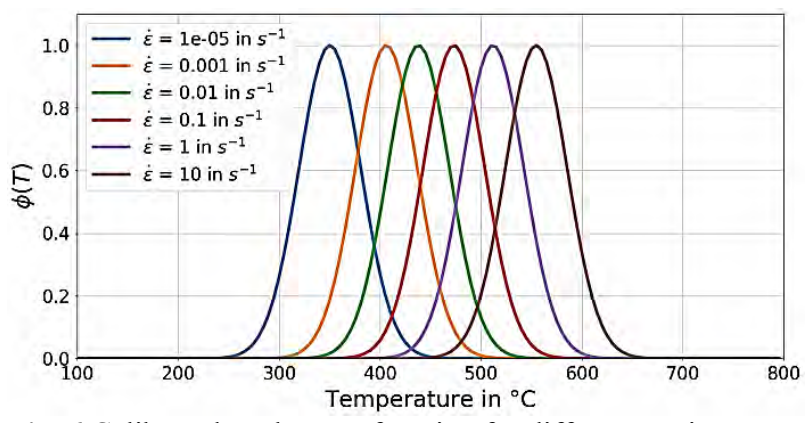

Fig. 6 Calibrated IE damage function for different strain rates.

Introducing the IE damage factor from Eq. (25) in Eq. (22) one obtains the desired expression:

$$
\frac{d a}{d N_{\text {tot }}}=\left(1+\frac{l}{\lambda} \varphi(T)\right) * \beta\left(d_{N^{\prime}} D_{T M F} a\right)^{B} .
$$

In Eq. (27) the IE damage is only active if $\varphi(T)>0$, and it leads to an acceleration of crack propagation within the temperatures affected by intergranular embrittlement.

By integrating the damage over the crack length for an initial crack size $a_{0}$ (i.e. grain size) and a technical crack size $a_{f}$, the number of cycles consumed by fatigue damage including intergranular damage results in:

$$
N_{t o t}=\frac{1}{\left(1+\frac{l}{\lambda} \varphi(T)\right)} * \frac{a_{f}^{1-B}-a_{0}^{1-B}}{\beta *(1-B)} *\left(d_{N^{\prime}} D_{T M F}\right)^{-B} .
$$

Rearranging the terms in Eq. (27) the crack propagation per cycle could be rewritten as:

$$
\frac{d a}{d N_{t o t}}=\beta_{I E}(T) *\left(d_{N^{\prime}} D_{T M F} a\right)^{B},
$$

where:

$$
\beta_{I E}(T)=\beta *\left(1+\frac{l}{\lambda} \varphi(T)\right) .
$$

This leads to a more compact expression for Eq. (28):

$$
N_{t o t}=\frac{a_{f}{ }^{1-B}-a_{0}{ }^{1-B}}{\beta_{I E}(T) *(1-B)} *\left(d_{N^{\prime}} D_{T M F}\right)^{-B} .
$$

The above generalized formulation of the lifetime model enables lifetime assessment of a material presenting time dependent behaviour and IE damage, however, it can also be applied for less complex material behaviours by deactivating the time dependency in case of an elastoplastic material behaviour or by deactivating the IE damage, for materials were there is no ductility drop at the IE temperature. 
Table 1 gives an overview of the model configuration for different material behaviours:

Table 1. Overview of the generalized lifetime model

\begin{tabular}{|c|c|c|}
\hline $\begin{array}{c}\text { Material } \\
\text { behaviour }\end{array}$ & $\begin{array}{l}\text { Generalized lifetime } \\
\text { model configuration }\end{array}$ & $\begin{array}{c}\text { Individualised } \\
\text { lifetime } \\
\text { formulation }\end{array}$ \\
\hline $\begin{array}{c}\text { Elasto-visco- } \\
\text { plastic } \\
\text { IE damage } \\
\end{array}$ & $\begin{array}{c}\beta_{\mathrm{IE}}(\mathrm{T})=\beta_{\mathrm{IE}}(\mathrm{T}) ; \\
\varphi(\mathrm{T})>0 \\
\mathrm{D}_{\mathrm{TMF}}=\frac{\mathrm{Z}_{\mathrm{D}}}{\sigma_{\mathrm{cy}}} \mathrm{F}(\mathrm{t}, \sigma, \mathrm{T})\end{array}$ & $\begin{array}{c}\mathrm{N}_{\text {tot }}=\frac{\mathrm{a}_{\mathrm{f}}{ }^{1-\mathrm{B}}-\mathrm{a}_{0}{ }^{1-\mathrm{B}}}{\beta_{\mathrm{IE}}(\mathrm{T}) *(1-\mathrm{B})} * \\
\quad\left(\mathrm{~d}_{\mathrm{N}^{\prime}} \mathrm{D}_{\mathrm{TMF}}\right)^{-\mathrm{B}},\end{array}$ \\
\hline $\begin{array}{c}\text { Elasto-visco- } \\
\text { plastic } \\
\text { No IE } \\
\text { damage }\end{array}$ & $\begin{aligned} \beta_{\mathrm{IE}}(\mathrm{T}) & =\beta ; \varphi(\mathrm{T})=0 \\
\mathrm{D}_{\mathrm{TMF}} & =\frac{\mathrm{Z}_{\mathrm{D}}}{\sigma_{\mathrm{cy}}} \mathrm{F}(\mathrm{t}, \sigma, \mathrm{T})\end{aligned}$ & $\begin{array}{c}\mathrm{N}_{\text {tot }}=\frac{\mathrm{af}_{\mathrm{f}}{ }^{1-\mathrm{B}}-\mathrm{a}_{0}{ }^{1-\mathrm{B}}}{\beta *(1-\mathrm{B})} * \\
\left(\mathrm{~d}_{\mathrm{N}^{\prime}} \mathrm{D}_{\mathrm{TMF}}\right)^{-\mathrm{B}}\end{array}$ \\
\hline $\begin{array}{l}\text { Elasto-plastic } \\
\text { IE damage }\end{array}$ & $\begin{array}{c}\beta_{\mathrm{IE}}(\mathrm{T})=\beta_{\mathrm{IE}}(\mathrm{T}) ; \\
\varphi(\mathrm{T})>0 \\
\mathrm{D}_{\mathrm{TMF}}=\frac{\mathrm{z}_{\mathrm{D}}}{\sigma_{\mathrm{cy}}} ; \mathrm{F}(\mathrm{t}, \sigma, \mathrm{T})= \\
1\end{array}$ & $\begin{array}{c}\mathrm{N}_{\text {tot }}=\frac{\mathrm{a}_{\mathrm{f}}{ }^{1-\mathrm{B}}-\mathrm{a}_{0}{ }^{1-\mathrm{B}}}{\beta_{\mathrm{IE}}(\mathrm{T}) *(1-\mathrm{B})} * \\
\left(\mathrm{~d}_{\mathrm{N}^{\prime}} \frac{\mathrm{Z}_{\mathrm{D}}}{\sigma_{\mathrm{cy}}}\right)^{-\mathrm{B}},\end{array}$ \\
\hline $\begin{array}{c}\text { Elasto-plastic } \\
\text { No IE } \\
\text { damage }\end{array}$ & $\begin{array}{c}\beta_{\mathrm{IE}}(\mathrm{T})=\beta ; \varphi(\mathrm{T})=0 \\
\mathrm{D}_{\mathrm{TMF}}=\frac{\mathrm{Z}_{\mathrm{D}}}{\sigma_{\mathrm{cy}}} ; \mathrm{F}(\mathrm{t}, \sigma, \mathrm{T})= \\
1\end{array}$ & $\begin{array}{c}\mathrm{N}_{\text {tot }}=\frac{\mathrm{a}_{\mathrm{f}}{ }^{1-\mathrm{B}}-\mathrm{a}_{0}{ }^{1-\mathrm{B}}}{\beta *(1-\mathrm{B})} * \\
\left(\mathrm{~d}_{\mathrm{N}^{\prime}} \frac{\mathrm{Z}_{\mathrm{D}}}{\sigma_{\mathrm{cy}}}\right)^{-\mathrm{B}},\end{array}$ \\
\hline
\end{tabular}

Comparing Eq.(31) with Eq.(23) one observes that the effect of IE damage can be included in the lifetime model by just introducing a temperature dependency in the prefactor $\beta$.

In the selected material neither time dependent behaviour, nor oxidation effects are observed within the studied temperature range $\left(\mathrm{RT}-500^{\circ} \mathrm{C}\right)$. For this reason, the function $F(t, \sigma, T)=1$ and the $D_{T M F}$ parameter reduces itself basically to the Heitmann parameter. Therefore, the lifetime model for the investigated GJS can be written as:

$$
N_{t o t}=\frac{a_{f}{ }^{1-B}-a_{0}{ }^{1-B}}{\beta_{I E}(T) *(1-B)} *\left(\frac{Z_{D}}{\sigma_{c y}}\right)^{-B} .
$$

Where $\beta$ and $\mathrm{B}$ are material parameters to be fitted in absence of IE, i.e. at RT. To this end, a more comfortable way of writing Eq. (32) for the parameters calibration at RT would be the following:

$$
N_{t o t}=A_{R T} *\left(\frac{Z_{D}}{\sigma_{c y}}\right)^{-B}
$$

Rearranging Eq. (33) and linearizing the terms of the expression results in Eq. (34):

$$
\log \left(\frac{Z_{D}}{\sigma_{c y}}\right)=\log \left(\frac{1}{A_{R T}}\right)^{B}+B * \log \left(N_{t o t}\right) .
$$

This small reformulation of the Eq. (33) enables to obtain the material parameters $\mathrm{B}$ and $\mathrm{A}_{\mathrm{RT}}$ directly from a Least Squares linear fit to fatigue data at RT. The fit of the slope leads to the value of parameter $\mathrm{B}$ and from the intercept value of the fit, $A_{\mathrm{RT}}$ can be obtained. Then, $\beta$ can be calculated as:

$$
\beta=\frac{a_{f}^{1-B}-a_{0}{ }^{1-B}}{A_{R T} *(1-B)}
$$

with a technical final crack length $\mathrm{a}_{\mathrm{f}}=1000 \mu \mathrm{m}$ and an initial defect size $\mathrm{a}_{0}=100 \mu \mathrm{m}$.

By substitution of the adjusted material parameters in Eq. (32), it is now possible to calculate a predicted lifetime for each one of the available fatigue tests.

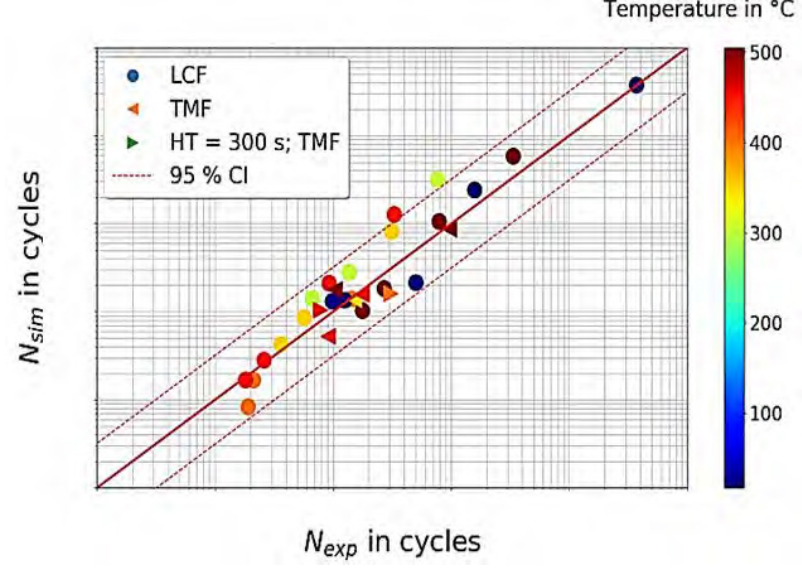

Fig. 7. Predicted lifetime vs experimental lifetime.

The quality of the model is illustrated by Fig. 7, where the predicted lifetime by the calibrated model is plotted against the corresponding experimental lifetime for every performed isothermal LCF test and OPTMF test.

\subsubsection{Lifetime model validation and probabilistic interpretation of lifetime scatter}

Assuming the proposed model is able to reproduce the damage mechanisms responsible for reducing the lifetime of GJS at intermediate temperatures, one can now interpret the scatter in Fig. 7 in terms of a defect distribution.

In Eq. (32) a fixed initial defect size was selected in order to integrate the lifetime. However the size of the initial defect is not equal for every test specimen. Material defects are usually represented by a probability distribution function (normal, Weibull, logarithmic...). The best way of determining distributions of defects serving as crack starters (i.e. graphite nodules, pores, shrinkage cavities) is by image processing of metallographic samples. Unfortunately this method is still very time and cost extensive, since casting defects are difficult to detect automatically within a fracture surface. In this section an indirect way of inferring the representative defect distribution probability density function is introduced.

The indirect inferred defect size was calculated for every test specimen by drawing a line of slope -B passing through every single experiment when plotting $\frac{Z_{D}}{\sigma_{c y}}$ versus the experimental lifetime. Thereby, an intercept value is obtained for every test. From the intercept value, it is possible to calculate this fictive initial defect size by simply solving for the value of $\mathrm{a}_{0}$ in Eq. (32):

$$
a_{0, s i m, i}=a_{f}-\left[\beta_{I E}(T) * A_{i} *(1-B)\right]^{\frac{1}{1-B}},
$$

where the index " $i$ " represents one tested specimen. $A_{i}$ is derived from its respective intercept value for every test in a similar manner $A_{\mathrm{RT}}$ was determined in the previous section. Note, that for this calculation $\beta_{\mathrm{IE}}(\mathrm{T})$ and not $\beta$ must be used otherwise the initial defect size would be calculated without accounting for IE damage and would 
lead to overestimated initial defect sizes for the temperatures were this mechanism is active.

The inferred initial defect sizes are shown in the histogram from Fig. 8. A 3-parameter Weibull distribution function (PDF) was fitted in order to reproduce the distribution of the initial size defects. In Fig. 9 the cumulative distribution function (CDF) based on the fitted 3-parameter distribution function is plotted against the empirical cumulative probability distribution of the initial defect size.

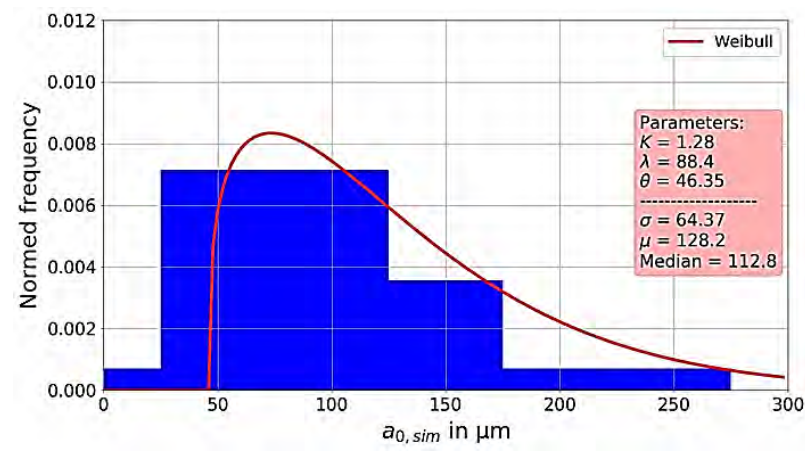

Fig. 8. PDF of the fictive initial defect size.

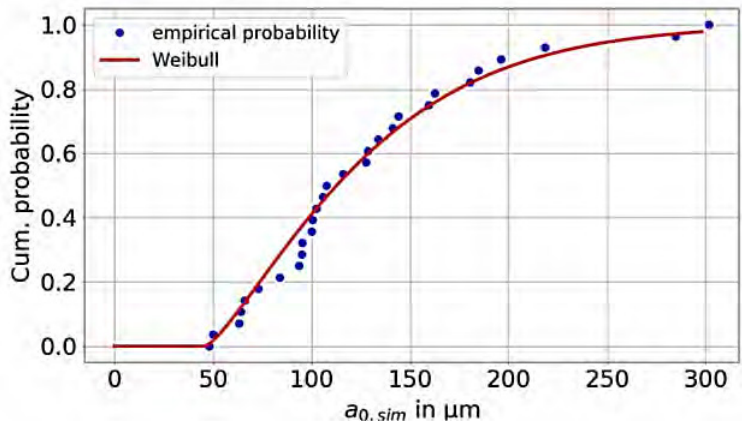

Fig. 9. CDF of the fictive defect size.

Figs. 10-16 illustrate the simulated Wöhler curves for different initial flaw size probabilities. For each selected probability: $\mathrm{P}=0.95, \mathrm{P}=0.5$ and $\mathrm{P}=0.05$, its corresponding initial defect size was extracted from the Weibull cumulative distribution function in Fig. 9. For each obtained initial defect the lifetime was newly integrated leading to a new point in the Wöhler diagram. This process was repeated for every test specimen and for each studied temperature a Wöhler diagram was generated.

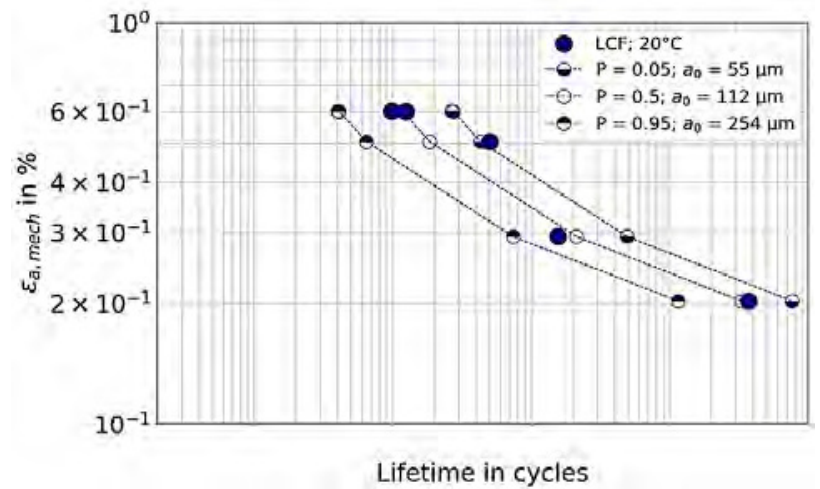

Fig. 10. Predicted vs experimental lifetimes at RT.

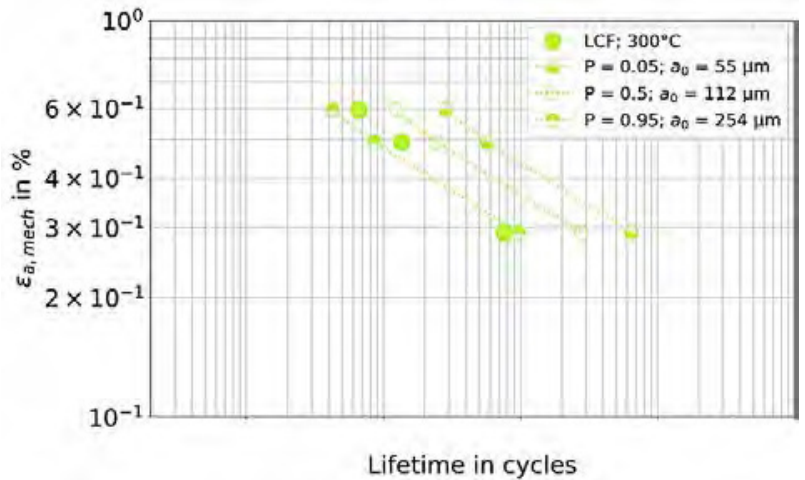

Fig. 11. Predicted vs experimental lifetimes at $300^{\circ} \mathrm{C}$.

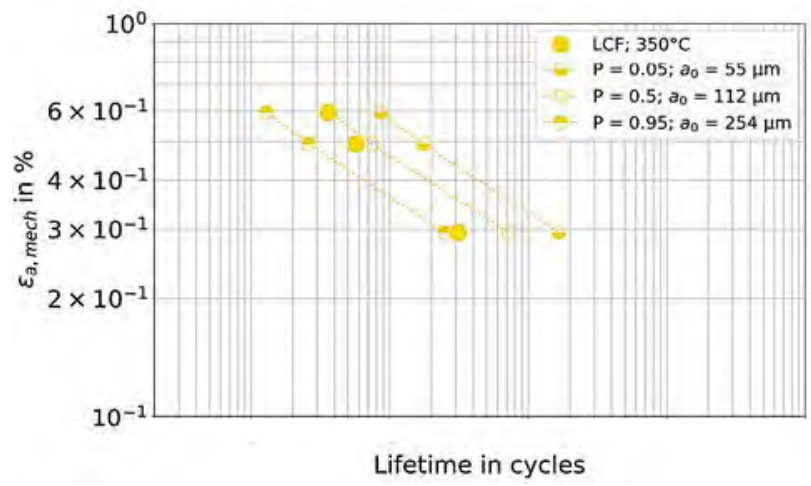

Fig. 12. Predicted vs experimental lifetimes at $350{ }^{\circ} \mathrm{C}$.

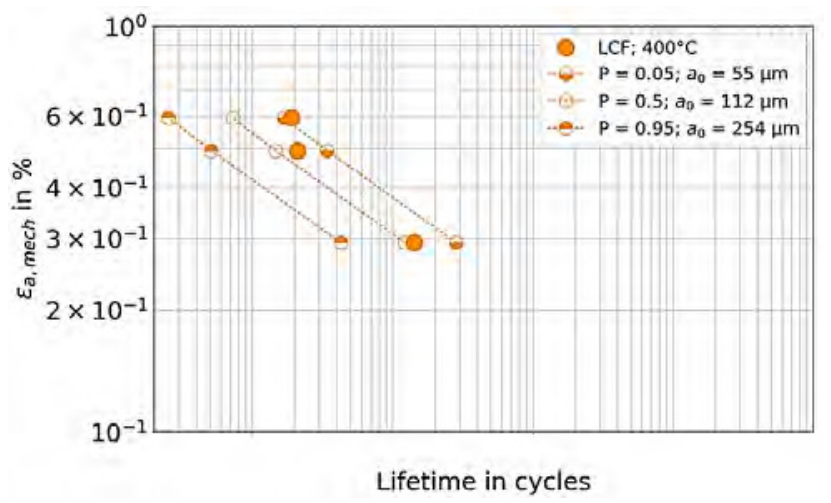

Fig. 13. Predicted vs experimental lifetimes at $400{ }^{\circ} \mathrm{C}$.

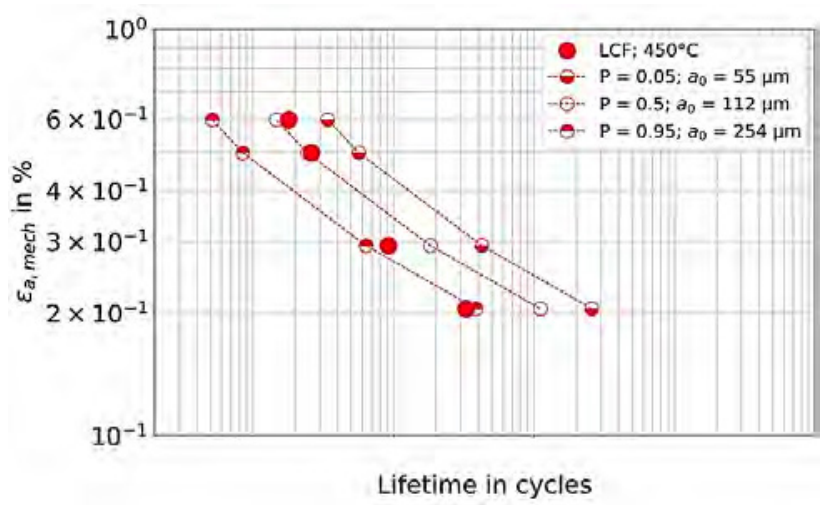

Fig. 14. Predicted vs experimental lifetimes at $450^{\circ} \mathrm{C}$. 


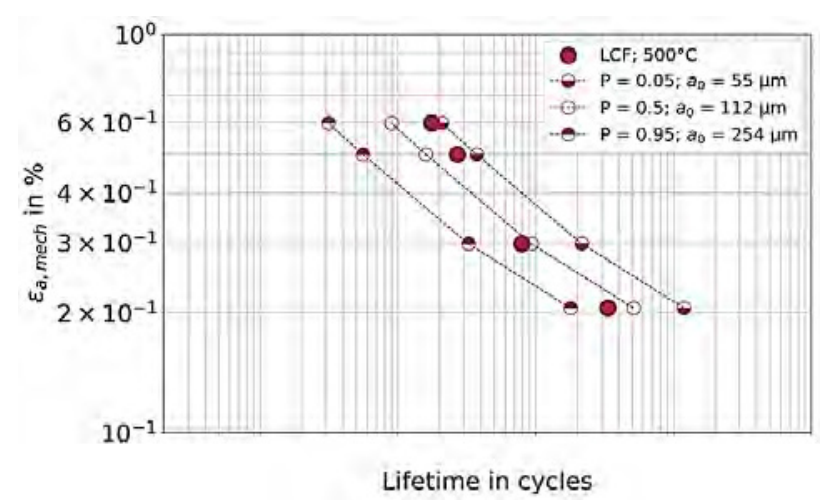

Fig. 15. Predicted vs experimental lifetimes at $500{ }^{\circ} \mathrm{C}$.

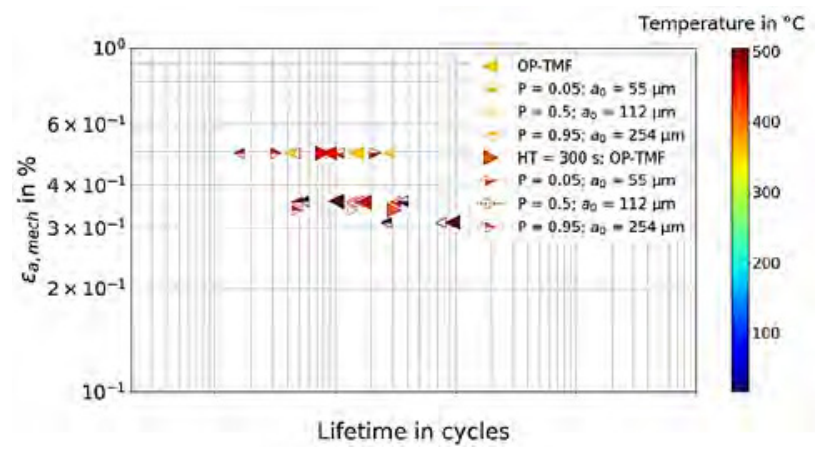

Fig. 16. Predicted vs experimental lifetimes for OPTMFs.

\section{Summary}

In this work it was assumed, that intergranular embrittlement is responsible for the observed lifetime reduction of LCF tests conducted at $400{ }^{\circ} \mathrm{C}$ and $450{ }^{\circ} \mathrm{C}$ and the ductility drop of tensile tests nearby $400{ }^{\circ} \mathrm{C}$. A lifetime model based on the $\mathrm{D}_{\mathrm{TMF}}$ damage parameter including intergranular embrittlement damage was formulated and calibrated. The predicted lifetimes are in very good agreement with the corresponding experimental lifetimes.

Further investigations are required in order to gain understanding about the process leading to IE damage at temperatures nearby $400^{\circ} \mathrm{C}$ and the strain rate dependency of the prefactor $\beta$ from the crack growth law. To this end, fatigue crack growth tests should be carried out within the studied temperature range.

Due to the typical variability of defect size in DCIs materials (shrinkage cavities, pores or graphite size) a probabilistic assessment of the initial defect size was included, allowing a deterministic-probabilistic interpretation of the scatter from the experimental lifetimes.

ACKNOWLEDGEMENTS: This project has received funding from the European Union's Horizon 2020 research and innovation programme under grant agreement No 634135 .

\section{References}

[1] T. Seifert, H. Riedel, "Fatigue Life Prediction of High Temperature Components in Combustion Engines and Exhaust Systems," EASC (2009)
[2] F. T. Shiao, T. S. Lui, L. H. Chen, and S. F. Chen, "Eutectic cell wall morphology and tensile embrittlement in ferritic spheroidal graphite cast iron," Metall. Mater. Trans. A, vol. 30, no. July, pp. 1775-1784 (1999).

[3] H.-M. Lin, T.-S. Lui, and L.-H. Chen, "Effect of Silicon Content on Intergranular Embrittlement of Ferritic Spheroidal Graphite Cast Iron Suffered from Cyclic Heating.," Mater. Trans., vol. 44, no. 1, pp. 173180 (2003)

[4] H.-M. Lin, T.-S. Lui, and L.-H. Chen, "Effect of Silicon Content on Intergranular Embrittlement of Ferritic Spheroidal Graphite Cast Iron Suffered from Cyclic Heating.," Mater. Trans., vol. 44, no. 1, pp. 173180 (2003)

[5] S. F. Chen, T. S. Lui, and L. H. Chen, "The effect of phosphorus segregation on the intermediate-temperature embrittlement of ferritic, spheroidal graphite cast iron," Metall. Mater. Trans. A, vol. 25, no. 3, pp. 557-561 (1994)

[6] X. Wu, G. Quan, R. MacNeil, Z. Zhang, and C. Sloss, "Failure Mechanisms and Damage Model of Ductile Cast Iron Under Low-Cycle Fatigue Conditions," Metall. Mater. Trans. A, vol. 45, no. 11, pp. 5085-5097 (2014)

[7] T. Kobayashi and K. Nishino, "Embrittlement of Ferritic Spheroidal Graphite Cast Iron by Magnesium," J. Japan Foundry Eng. Soc., vol. 70, p. .273-278 (1998)

[8] P. H. Riedel, "Fracture at high temperatures", Materials Research and Engineering. Springer, Berlin, Heidelberg (1987)

[9] V. Schivje, J, "Some formulas for the crack opening stress level“, Eng. Fracture Mechanics., 14, 461-465 (1981)

[10] C.F. Shih, Tables of Hutchinson-Rice-Rosengren singular field quantities, Tech. rep. Brown University Report MRL E-147 (1983)

[11] H. H. Heitmann, H. Vehoff, and P. Neumann, "Life prediction for random load fatigue based on the growth behavior of microcracks," in Advances in fracture research 84 - proceedings of ICF6, pp. 3599-3606 (1984)

[12] W. Schmitt, R. Mohrmann, H. Riedel, A. Dietsche, A. Fischersworring-Bunk, Modelling the fatigue life of automobile components, In: Fatigue 2002 - Proceedings of the Eight International Fatigue Congress held 3-7 June 2002, Stockholm, Sweden (Ed. A.F. Blom), 781-788 (2002)

[13] J. C. Newman, A crack opening stress equation for fatigue crack growth, Int. J. Fract., Vol. 24, 131-135 (1984)

[14] T. S. Lui and C. G. Chao, "High-temperature properties of ferritic spheroidal graphite cast iron," J. Mater. Sci., vol. 24, no. 7, pp. 2503-2507 (1989) 